1 The inconvenient truth about convenience polyandry

2

3 Rebecca A. Boulton ${ }^{1,2,3}$, Marlene Zuk ${ }^{4}$, and David M. Shuker ${ }^{3}$

$4{ }^{1}$ College of Life and Environmental Sciences, University of Exeter, Cornwall, TR10

5 9FE, UK

62 Department of Entomology, University of Minnesota, St Paul, MN 55108, USA

$7 \quad{ }^{3}$ School of Biology, University of St Andrews, St Andrews, KY16 9TH, UK

$8{ }^{4}$ Department of Ecology, Evolution and Behavior, University of Minnesota, St Paul,

$9 \quad$ MN 55108, USA

10

11 Corresponding author: Boulton, R.A. (r.boulton@exeter.ac.uk)

12

13 Keywords: Convenience polyandry, sexual harassment, sexual conflict, mating

14 systems

15

16

17

18

19

20

21

22

23

24 
Abstract

In the last two decades molecular techniques have revealed that polyandry, or multiple mating by females with different males, is common. One explanation is that females engage in convenience polyandry, mating multiply to reduce the costs of sexual harassment. Although the underlying logic of convenience polyandry is clear, and harassment often seems to influence mating outcomes, it has not been subjected to as thorough theoretical or empirical attention as other explanations for polyandry. Here, we re-examine convenience polyandry in the light of a new generation of studies showing previously unconsidered benefits of polyandry. We suggest that true convenience polyandry is likely to be a fleeting phenomenon, but potential feedback loops between resistance to males and the costs and benefits of mating.

The history of polyandry

Mating systems are often classified as either monogamous, with a single male paired with a single female, or polygamous, with multiple mates of one or the other sex. Polygamy in turn can be subdivided into polygyny (one male with multiple females) or polyandry (one female with multiple males; see glossary). The near ubiquitous nature of polygyny has never been debated, but polyandry has had a somewhat contradictory history. On the one hand, researchers using insects and other invertebrates as study organisms have often acknowledged the prevalence of 
multiple mating by females [1]. In vertebrates, however, polyandry has been seen to

require pair bond formation between one female and multiple males, with

51 commonly cited examples including some wading birds, pipefish and dendrobatid frogs. Such long-term associations are rare in vertebrates, and hence polyandry was viewed as an anomaly, with the rationale being that a female could be fully inseminated by a single male, making multiple mating with different males unnecessary. These taxon-wide generalities led to inherent contradictions running through the two streams of literature, with polyandry either being viewed more as the norm (in insects [2]) or a rare evolutionary exception (in birds and mammals [3]).

In the last two decades, however, advances in molecular techniques for assaying paternity have resulted in a more integrated perception of female mating behaviour. Multiple mating and extra-pair copulations by females are all-but ubiquitous, even in species once thought to be strictly monogamous [4]. Polyandry is no longer regarded as a rare phenomenon, involving, for example, sex role reversal and paternal care in the vertebrates, but as a common mating system where females engage in copulations with multiple partners [4-5]. This conceptual shift has led to the 'polyandry revolution' [5] - a surge in studies seeking to untangle the myriad evolutionary causes and consequences of female multiple mating. As a result, the reasons that females engage in polyandry have been widely discussed [5], bringing a welcome expansion of mating system theory.

71 Importantly, the idea that females engage in polyandry not to gain benefits, but rather to limit the costs imposed upon them by harassing males, has been discussed 
throughout the polyandry revolution. This hypothesis is known as convenience

polyandry [2], and while it continues to be highlighted by those seeking to understand why females engage in multiple matings [for recent papers see: 6-17], we argue that it would benefit from more rigorous theoretical and empirical testing that other, benefits-driven hypotheses for the evolution of polyandry, have been subject [16-22].

The polyandry revolution has demonstrated that females are by no means passive players in sexually antagonistic co-evolution [23-24] and if they are able to escape harassment in less costly ways than by mating, then they will [25-27]. What is more, if indirect genetic benefits invariably follow from polyandry [17], such convenience polyandry, where the only benefit is cost mitigation, might be rare and fleeting. In this review, we consider recent theoretical and empirical developments in polyandry research in order to critically reappraise the convenience polyandry hypothesis.

\section{What is convenience polyandry?}

The benefits of coercive strategies to males are relatively clear, but female strategies

91 for curtailing the costs of harassment are more ambiguous [6]. Convenience polyandry is a commonly cited example of such a strategy. The traditional definition of convenience polyandry is that females accept superfluous matings to reduce the convenience polyandry as follows: 
Convenience polyandry occurs when females increase their receptivity to mating based on the relative costs of resistance and mating, such that they are more likely to mate when the costs of resistance or avoidance exceed the net costs of mating.

The convenience polyandry hypothesis is intuitive - attempting to resist superfluous matings with persistent males can be more energetically demanding, can reduce time available for other necessary activities (foraging, ovipositing, etc.) and can even result in more physical damage (including death), than accepting the mating (see Figure 1). Although some good examples of convenience polyandry exist in nature

107 (see Box 1), the hypothesis has not been elaborated upon theoretically (even though 108 the coevolutionary consequences of sexual harassment and resistance have been extensively modelled, these dynamics are not equivalent to the convenience

110 polyandry hypothesis). Moreover, when convenience polyandry is typically

111 described, it is usually treated as separate from direct or indirect benefits 112 explanations for female multiple mating.

115 Likewise, convenience polyandry is only occasionally empirically tested, and tends to 116 be inferred from observations of high female mating rates at high male densities [10; 117 15; 31]. This interpretation is based on the idea that more frequent encounters with 118 males result in elevated resistance costs for females, but the relative costs of 119 resistance compared to mating are seldom separated or measured explicitly (and see 120 [32] for an outstanding example). The logic of convenience polyandry is compelling, 
121 but we still need to measure resistance costs and map them to different behavioural

122 or ecological contexts in order to understand why the female mating rate is subject

123 to evolutionary change. We suggest that convenience polyandry should only be

124 ascribed when the costs of resistance have been shown to exceed the net costs of

125 mating, such that there is strong evidence for cost mitigation. Below we describe

126 new findings in support of our contention, and their implications for convenience

127 polyandry, which we use to suggest a framework in which to test it (see Table 1).

129 What if polyandry is the null hypothesis?

130

131 Despite, or perhaps because of, the ubiquity of polyandry, active female multiple

132 mating, as opposed to passive acceptance of matings in the face of sexual

133 harassment, is sometimes regarded as an anomaly that requires an explanation [7].

134 It has been suggested that societal biases regarding appropriate or expected female

135 behaviour might have a role to play in such interpretations (42-43; see Box 2).

136 However, this idea is also based on the founding principles of sexual selection

137 theory. Bateman's principles, based on work in Drosophila melanogaster, state that

138 females gain less reproductive success per mating than males [44], and so high levels

139 of polyandry are not expected. Although the robustness and applicability of

140 Bateman's conclusions have been called into question [see 43; 45-46], the general

141 acceptance of this paradigm (which may itself have a socio-cultural component; 42;

142 see also 47) has meant that the evolutionary significance of polyandry was, until

143 relatively recently, overlooked. 
146 The polyandry revolution, however, has shown that monandry is rare compared to

147 polyandry, and recent work has demonstrated, both theoretically and empirically,

148 that there are many, less clear-cut reasons for females to engage in polyandry [7; 9;

149 19-22; 33; 49-57]. Moreover, the idea that polyandry is unexpected, and the implicit

150 treatment of monandry (and by extension convenience polyandry), as the null

151 hypothesis has been increasingly called into question, perhaps most explicitly by

152 Kokko \& Mappes ([33] see Figure 2). Using a modelling approach, they found that

153 even when mating carried fecundity or longevity costs, polyandry was predicted to

154 predominate over monandry. A key assumption in their models was that female

155 receptivity was consistent for each encounter with a male, such that the probability

156 of acceptance did not change over a female's lifetime. Thus the risk of dying a virgin,

157 combined with a lack of information about future encounters, made polyandry the

158 most likely strategy, even if the optimal life history strategy under perfect

159 knowledge and free access to mates was monandry.

160

161

162 What is more, other bet-hedging strategies that promote genetic diversity [17] and

163 genetic compatibility [49], or ensure fertility under inbreeding [49] or outbreeding

164 [9], might mean that polyandry is the optimal strategy for an individual to adopt

165 because it spreads the risk of mating failure [19; 33; 51-52]. Moreover, polyandry

166 can be advantageous to populations, for instance reducing extinction risk by purging

167 mutation load, increasing effective population size [53] and rescuing populations

168 from the effects of inbreeding [22]. Indeed, the importance of polyandry in 
preventing extinction has been demonstrated in populations of Drosophila

pseudobscura harbouring a selfish X-linked drive [54].

172 These alternative functions for polyandry mean that it might be selectively favoured

173 even if it does not maximise the fitness of individual females under all

174 circumstances, because it can minimize the risk of mating failure. Under bet-hedging, 175 a monandrous female might have very high fitness (if mating is costly) but the risk of 176 mating failure (either because she fails to mate entirely or does not mate with a 177 compatible male) means that she could have zero fitness [19; $33 ; 51-52 ; 55]$. A 178 polyandrous female, on the other hand, might never experience such high fitness (if 179 mating is costly), but the risk of complete reproductive failure is lower. In this way, 180 polyandry might persist in the absence of individual benefits, and even when it is 181 costly to females (see $[19 ; 51-52 ; 58-59]$ for more on bet-hedging and polyandry).

183 If polyandry arises because of a baseline level of receptivity that ensures fertility, but

184 leads to multiple mating across the population, then we should re-evaluate the 185 convenience polyandry hypothesis. First, the absence of benefits can no longer be 186 taken as suggestive of convenience polyandry, because polyandry is just the null expectation. Second, convenience polyandry can no longer be inferred from

188 increased female mating rates when the sex ratio is more male-biased, as Kokko \& 189 Mappes [33] demonstrate. To counter the risk of mating failure, if females are 190 equally receptive to every mating attempt, then this means that they will mate with 191 a set proportion of all males they encounter. The outcome is that they will mate 

convenience polyandry. If, on the other hand, polyandry evolves because it is

213 otherwise beneficial (such that the costs of mating relative to resistance are 214

more times as the sex ratio becomes more male-biased, without necessarily changing their receptivity to each mating (Figure 2; Table 1, criterion 1).

If convenience polyandry is not necessarily an appropriate conclusion to draw when females mate multiply without obvious benefits, when is it likely to occur, and how can we test it empirically (Table 1)? Changes in the female mating rate under different sex ratio regimes alone do not provide enough data to conclude that convenience polyandry is occurring, as discussed above. If, however, females accept a greater proportion of mating attempts when the sex ratio becomes more malebiased, a case can be made for convenience polyandry because this suggests that females alter their receptivity to mating based on the level of harassment they experience (criterion 1, Table 1).

\section{The causes of convenience}

Polyandry is convenient when resisting copulation is more costly than acceptance, and when females act to mitigate the costs of resistance by altering their propensity to mate. We emphasise that focusing on the costs of resistance is key to determining whether polyandry is convenient. If multiple mating has been selectively favoured to reduce resistance costs, then it is convenient under the usual definitions of reduced; Table 1, Figure 1) then it is not convenient in the sense implicit in the 
definition of convenience polyandry. In other words, it is not "true" convenience

216 polyandry.

218 In many situations, we expect that females will act to reduce the costs of resistance.

219 For example, females might gain protection from male mate guarding, which allows

220 them to forage or oviposit uninterrupted. In other situations, male harassment

221 might result in significant harm or even death, again making resistance not

222 worthwhile (Box 1). However, if we consider that mating is not the only way that

223 females can limit harm induced by harassing males, then to demonstrate

224 convenience polyandry it is necessary to test if (and when) it is more costly for a

225 female to resist a mating than it is to accept it (criterion 2; Table 1). Alternatives to

226 acquiescing to mating can include crypsis and androgyny, as seen in damselflies [25-

227 26] or hiding, as seen in water striders [27].

228

229 One method that has been used to test whether the costs of resistance (CR) exceed

230 the costs of mating (CM; criterion 2) is by using ablation studies. In these studies,

231 male genitalia are ablated so that they are unable to mate with females but can still

232 harass them. If $\mathrm{CR}>\mathrm{CM}$ then females housed with ablated males will suffer greater

233 fitness costs than females housed with intact males. The ability to mate will allow

234 females to mitigate the costs of resistance by accepting the (lower) costs of mating

235 [36; see 60 for an elegant example of how female resistance can be manipulated

236 without male ablation]. 
Despite the clear and intuitive nature of the ablation test, we know of no study that

239 has shown the costs of resistance exceed the costs of mating (Table 1, criterion 2).

240 Some of the apparent ambiguities from ablation studies (Table 1, criterion 2) might

241 occur as a result of environmental effects on the costs of resistance and mating. For

242 instance, environmental context might render polyandry convenient in some

243 situations but otherwise beneficial in others. If, for example, the key cost of

244 resistance is reduced foraging efficiency, we should compare females that have easy

245 access to abundant food resources with those that do not when we wish to see if

246 there is scope for convenience polyandry to reduce costs of resistance (criterion 3).

248 More generally, context is key to convenience. Before convenience polyandry can be

249 tested, it is important to identify and measure the costs of mating in the absence of

250 harassment in a variety of ecologically appropriate contexts [see 61-62]. The results

251 of such studies can then be used to design experiments, including ablation studies,

252 to measure the relative costs of mating and resistance (criterion 2), and how females

253 respond to these in terms of their propensity to re-mate (criterion 3). Ultimately

254 these results could inform experimental evolution studies that manipulate the

255 environment to alter the relative costs of mating and resistance. Such studies would

256 allow assessment of the selective potency of the benefits of mating vs the costs of

257 resistance in driving evolutionary changes in the female mating rate (Table 1 ,

258 criterion 3$)$. 
262 Even when females do increase their receptivity to reduce the costs of resistance,

263 polyandry might not remain convenient for long. When females engage in polyandry

264 they might alter the other costs and benefits associated with mating in a number of

265 ways, which we outline below. Importantly this can change whether polyandry is a

266 matter of convenience, necessity or gain (Figure 1).

267

268

Indirect genetic benefits

270 Females can benefit from polyandry via the post-copulatory sexual selection that it

271 elicits. By mating multiply, whether for convenience or not, females elicit sperm

272 competition. As a result, their offspring are more likely to be sired by males that

273 succeed in sperm competition, potentially resulting in indirect genetic benefits,

274 including good genes [56] and Fisherian 'sexy sperm' for their sons [57]. Even if such

275 benefits are weak $[18 ; 20]$, they can reduce the costs of mating relative to resistance

276 and could tip the balance, rendering polyandry more beneficial than it is convenient

277 (Figure 1). It is, however, worth noting that indirect costs could also occur in this

278 scenario through sexually antagonistic pleiotropy (i.e. polyandrous females

279 producing high fitness sons and low fitness daughters) which may counterbalance

280 these indirect benefits [63]).

Male harm

284 Another consequence of polyandry is that it induces sexual conflict over paternity, 285 which can favour chemical or physical male traits that harm females, reducing their 
receptivity to future matings, and providing males with greater paternity certainty

$287[24 ; 62]$. This too will alter the relative costs of mating vs resistance, in this case

288 increasing the costs of mating, which is expected to reduce the convenience of

289 polyandry (Figure 1).

290

291 Coercive 'sexy sons'

Convenience polyandry is selectively advantageous because it acts to reduce the

294 general costs associated with resistance (criterion 3) and as such females reduce

295 their reluctance to mate with any male (criterion 4). However, when females engage

296 in convenience polyandry, there is scope for sexual selection to arise through passive

297 mate choice (i.e. there is no requirement for active discrimination between, and

298 rejection of, several potential males). This could arise if females are more likely to

299 mate with males that can impose larger costs, such as larger males. We call this

300 Facultative Female Resistance (FFR, Table 1, Figure 1), to emphasise that female

301 resistance can vary with the male they are interacting with, on a male-by-male basis.

302 Importantly, by engaging in FFR, females can gain indirect genetic benefits through

303 passive mate choice (i.e. good genes and/or sexy 'coercive' sons). These benefits

304 then reduce the costs of mating compared to the costs of resistance and in turn shift

305 the selection on polyandry away from convenience (although again see [63] for an

306 example of a counter cost of polyandry in this context).

308 Water striders (Box 1) present a good example of how facultative female resistance

309 can render convenience polyandry beneficial in other ways, making it no longer 
strictly about reducing the costs of resistance. In many species of water strider,

311 males have non-intromittent genital claspers that allow them to endure female

312 struggles [28]. The larger these claspers, the better able males are to overcome such

313 struggles and successfully mate. Importantly, however, the size of these structures is

314 heritable [41], which means that by reducing their reluctance to mate with males

315 with large claspers, females can produce "sexy sons" that in turn will have higher

316 mating success (see Table 1, criterion 4).

Strategic ejaculate allocation

320 Perhaps most importantly, polyandry can induce positive feedback loops that lead to

321 its persistence despite non-negligible costs of mating [21; see Figure 1]. Such positive

322 feedback occurs because polyandry can result in sexual selection for smaller

323 ejaculates in males, reflecting the trade-off between partitioning ejaculates across

324 many matings versus increasing success in sperm competition over a few matings

325 [64-67]. When males either control mating or when polyandry is convenient, the

326 variance in the number of mates for a given female will increase. Under these

327 conditions, males will benefit from bet-hedging - spreading the risk of failing to sire

328 any offspring by investing more in mating with many females and transferring

329 smaller ejaculates to each of them [65-67].

331 Bocedi \& Reid [21] modelled this scenario and found that such strategic ejaculate

332 allocation increases the risk of sperm limitation for females and results in selection

333 on increased female mating frequency, even when mating is costly to females. These 
334 feedback loops were particularly potent, with a higher likelihood of evolving

335 polyandry, when sperm precedence was strong. When sperm precedence is strong, a

336 single male (usually the first or the last) will sire the majority of a female's offspring.

337 Under these conditions, investing in large, expensive ejaculates is far riskier than

338 investing in many smaller ejaculates because the likelihood of siring any offspring

339 from a given mating is lower than when sperm are mixed. Under strong sperm

340 precedence, selection will favour smaller ejaculates and subsequently increases

341 female mating rates to prevent sperm limitation. Empirical evidence for the

342 predictions of these models has been found, most notably across the bushcrickets

343 (Orthoptera: Tettigoniidae), where males in more polyandrous species have larger

344 testes but transfer smaller ejaculates to increase their relative paternity success

345 across matings [68-69, see also 70 for an excellent example of a bespoke model and

346 an empirical test of strategic ejaculate allocation theory].

348 Many of the processes outlined above will increase the optimal mating rate for

349 females by increasing the benefits of mating. In particular, if the risk of sperm

350 limitation is non-negligible, then polyandry becomes a matter of necessity rather

351 than convenience (Figure 1). The opportunity for these feedback loops to occur leads

352 us to suggest that true convenience polyandry might be a rather fleeting

353 phenomenon, because the consequences of polyandry (in terms of sexual conflict

354 and pre- and post-copulatory sexual selection) will almost always change the relative

355 costs of mating, and so polyandry may become advantageous independently of the

356 costs of resisting. Importantly, we are not suggesting that we no longer expect

357 females to attempt to reject some or all males, or that resistance to male 
harassment is no longer an important component of how mating behaviour evolves.

359 Rather, we wish to emphasise that selection on polyandry just to reduce mating

360 costs - the essence of convenience polyandry - may typically be only a transitory

361 phase in the overall evolutionary origin and maintenance of polyandry.

\section{Concluding Remarks}

Convenience polyandry requires that the costs of resistance exceed the costs of mating, for instance when male density is high or when males coerce females. It also requires that females alter their receptivity to mating in order to mitigate the costs

368 of resistance and not to gain other benefits. Whether polyandry can be considered convenient depends on whether selection favours polyandry because it mitigates a cost (resistance) or because it is otherwise beneficial. Some may argue that this is

371 purely semantic, but to understand closely associated evolutionary processes (such 372 as convenience vs benefits-driven polyandry) it is crucial to determine exactly how

373 selection acts and what it acts upon. Convenience polyandry - as generally accepted

374 - serves to reduce the costs of resistance, but once polyandry starts it can induce

375 other costs and benefits associated with mating such that it is no longer a matter of

376 convenience (Figure 1). As such, convenience polyandry may be a fleeting

377 phenomenon, but it could also be a phenomenon with the power to shape mating systems and patterns of sexual selection (Box 3).

380 Polyandry may be a matter of convenience under some situations, but within species 381 and populations selection for polyandry may differ according to the environment, 
female state, and the male's ability to overcome reluctance (see Box 3). To understand the evolutionary significance of cost mitigation in driving female mating

384 behaviour, researchers must explicitly test the convenience polyandry hypothesis, all 385 the while maintaining an awareness of potential socio-cultural biases about whether 386 or not we implicitly expect females to be monandrous or polyandrous (Box 2)

\section{Acknowledgements}

Thanks to Georgina Glaser, Ginny Greenway, Jessie Tanner, Justa Heinen-Kay, Lewis

Dean, Narmin Ghalichi, Rachel Olzer and Rebecca Ehrlich for their valuable input. We are also very grateful for the efforts of six anonymous reviewers who helped us clarify our thinking and the manuscript. Many thanks also to Locke Rowe for images.

395 R.A.B is supported by a grant from the International Community Fund (awarded by 396 the Leona M. and Harry B. Helmsley Charitable Trust).

\section{References}

399 [1] Boulton, R.A. and Shuker, D.M. (2013) Polyandry. Curr. Biol. 23, R1080R1081.

401 [2] Thornhill, R. and Alcock, J. (1983) The evolution of insect mating systems. Harvard University Press.

403 [3] Emlen, S.T. and Oring, L.W. (1977) Ecology, sexual selection, and the evolution of mating systems. Science. 197, 215-223. 
405 [4] Taylor, M.L. et al. (2014) Polyandry in nature: a global analysis. Trends. Ecol. Evol. 29, 376-383.

407 [5] Pizzari, T. and Wedell, N. (2013) Introduction: The polyandry revolution. Phil. Trans. R. Soc. B. Biol. Sci. 368,1-5.

409 [6] Snook, R.R. (2014) The evolution of polyandry. In The evolution of insect mating systems (Shuker, D.M. and Simmons, L.W., eds), pp. 59-180. Oxford University Press.

412 [7] Forstmeier, W. et al. (2014) Female extra-pair mating: adaptation or genetic constraint. Trends. Ecol. Evol. 29, 458-464

414 [8] Johannesson, K. et al. (2016) Non-random paternity of offspring in a highly promiscuous marine snail suggests postcopulatory sexual selection. Behav. Ecol. Sociobiol. 70, 1357-1366.

417 [9] Burdfield-Steel, E.R. et al. (2015) Do the benefits of polyandry scale with outbreeding? Behav. Ecol. 26, 1423-1431.

419 [10] Lee, P.L.M. et al. (2017) A review of patterns of multiple paternity across sea turtle rookeries. Adv. Mar. Biol. https://doi.org/10.1016/bs.amb.2017.09.004

421 [11] Lyons, K. et al. (2017) Who's my Daddy? Considerations for the influence of sexual selection on multiple paternity in elasmobranch mating systems. Ecol. Evol. 7, 5603-5612.

424 [12] McDonald, G.C. et al. (2017) Pre- and postcopulatory sexual selection favor aggressive, young males in polyandrous groups of red jungle fowl. Evolution. 71, 1653-1669. 
[13] Wells, C.P. et al. (2017). Determinants of multiple paternity in a fluctuating population of ground squirrels. Behav. Ecol. Sociobiol, DOI 10.1007/s00265017-2270-z

[14] Zhao, M. et al. (2016) Male pursuit of higher reproductive success drives female polyandry in the Omei treefrod. Anim. Behav. 111, 101-110.

[15] Pirog, A. et al. (2017) First evidence of multiple paternity in the bull shark (Carcharhinus leucas) Mar. Freshwater. Res. 68, 195-201.

[16] Anqvist, G. and Nilsson, T. (2000) The evolution of polyandry: multiple mating and female fitness in insects. Anim. Behav. 60, 145-164.

[17] Jennions, M.D. and Petrie, M. (2000) Why do females mate multiply? A review of the genetic benefits. Biol. Rev. 75, 21-64.

[18] Slatyer, R.A. et al. (2012) Estimating genetic benefits of polyandry from experimental studies: a meta-analysis. Biol. Rev. 87,1-33.

[19] Yasui, Y. and García-González, F. (2016) Bet-hedging as a mechanism for the evolution of polyandry, revisited. Evolution. 70, 385-397.

[20] Bocedi, G. and Reid, J.M. (2015) Evolution of female multiple mating: a quantitative model of the "sexually selected sperm" hypothesis. Evolution. 69, 39-58.

[21] Bocedi, G. and Reid, J.M. (2016) Coevolutionary feedbacks between female mating interval and male allocation to competing sperm traits can drive evolution of costly polyandry. Am. Nat. 187, 334-350.

[22] Bocedi, G. and Reid, J.M. (2017) Feed-backs among inbreeding, inbreeding depression in sperm traits, and sperm competition can drive evolution of costly polyandry. Evolution. 71, 2786-2802. 
451 [23] Perry, J.C. and Rowe, L. (2015) The evolution of sexually antagonistic phenotypes. Cold. Spring. Harb. Perspect. Biol., 7,017558.

453 [24] Dougherty, L.R. et al. (2017) Sexual conflict and correlated evolution between male persistence and female resistance traits in the seed beetle Callosobruchus maculatus. Proc. R. Soc. Lond. B. Biol. Sci. 284, 20170132.

456 [25] Outomuro, D. et al. (2016) Antagonistic natural and sexual selection on wing shape in a scrambling damselfly. Evolution. 70, 1582-1595.

458 [26] Galicia-Mendoza, I. et al. (2017) Male biased sex ratio reduces the fecundity of one of three female morphs in a polymorphic damselfly. Behav. Ecol. 28,

461 [27] Pineaux, M. and Turgeon, J (2017) Behavioural consistency in female 462 resistance to male harassment in a water strider species. Ethol. 123, 83-93.

463 [28] Rowe, L. et al. (1994) Sexual conflict and the evolutionary ecology of mating 464 patterns: water striders as a model system. Trends. Ecol. Evol. 9, 289-293.

465 [29] Han, C.S. and Jablonski, P.G. (2010) Male water striders attract predators to 466 intimidate females into copulation. Nat. Commun. 1, 1-6.

467 [30] Sih, A. and Krupa, J.J. (1992) Predation risk, food deprivation and non-random mating by size in the stream water strider, Aquarius remigis. Behav. Ecol. Sociobiol. 31, 51-56.

470 [31] Janowitz, S.A. and Fischer, K. (2012) Polyandry in Bicyclus anynana butterflies results from sexual conflict over mating. Ethol, 118, 1140-1148.

472 [32] Watson, P.J. et al. (1998). Sexual conflict and the energetic costs of mating 473 and mate choice in water striders. Am. Nat. 151, 46-58. 
474 [33] Kokko, H. and Mappes, J. (2013) Multiple mating by females is a natural outcome of a null model of mate encounters. Entomol. Exp. Appl. 146, 26-37.

[34] Fox, C.W. and Hickman, D.L. (1994) Influence of oviposition substrate on female receptivity to multiple mating in Callosobruchus maculatus (Coleoptera: Bruchidae) Ann. Entomol. Soc. Amer. 87, 395-398.

[35] den Hollander, M. and Gwynne, D.T. (2009) Female fitness consequences of male harassment and copulation in seed beetles, Callosobruchus maculatus. Anim. Behav. 78, 1061-1070.

[36] Harano, T. (2015) Receptive females mitigate costs of sexual conflict. J. Evol. Biol. 28, 320-327.

[37] Sakurai, G. and Kasuya, E. (2008). The costs of harassment in the adzuki bean 485 beetle. Anim. Behav. 75, 1367-1373.

486 [38] Harano, T. et al. (2006). Direct effects of polyandry on female fitness in Callosobruchus chinensis. Anim. Behav. 71, 539-548.

488 [39] McLain, D.K. et al. (2015) Size dependence of courtship effort may promote male choice and strong assortative mating in soldier beetles. Behav. Ecol. Sociobiol. 69, 883-894.

491 [40] Sniegula, S. et al. (2017) Do males with higher mating success invest more in armaments? An across-populations study in damselflies. Ecol. Entomol. 42, $526-530$.

494 [41] Perry, J.C. et al. (2017) The role of ecology, neutral processes and antagonistic coevolution in an apparent sexual arms race. Ecol. Lett. 20, 1107-1117. 
[42] Tang-Martinez (2016) Rethinking Bateman's Principles: Challenging Persistent Myths of Sexually Reluctant Females and Promiscuous Males. J. Sex. Res. 53, 532-559.

500 [43] Kokko, $\mathrm{H}$ (2017) Give one species the task to come up with a theory that spans them all: what good can come out of that? Proc. R. Soc. Lond. B. Biol. Sci. 284, 20171652.

503 [44] Bateman, A.J. (1948) Intra-sexual selection in Drosophila. Heredity. 2, 349-

504 368.

505 [45] Snyder, B.F. and Gowaty, P.A. (2007) A reappraisal of Bateman's classic study of intrasexual selection. Evolution. 61, 2457-2468.

507 [46] Gowaty, P.A. et al. (2012). No evidence of sexual selection in a repetition of Bateman's classic study of Drosophila melanogaster. Proc. Natl. Acad. Sci. U.S.A. $109,11740-11745$.

510 [47] Janicke, T. et al. (2016). Darwinian sex roles confirmed across animal kingdom. Sci. Adv. 10.1126/sciadv.1500983

512 [48] Dougherty, L.R. et al. (2013) Sexual stereotypes: the case of sexual 513 cannibalism. Anim. Behav, 313-322.

514 [49] Arbuthnott, D. et al. (2015) Female stick insects mate multiply to find compatible mates. Am. Nat. 186, 519-530.

516 [50] Bayoumy, M.H. et al. (2015) Polyandry restores female fertility and paternal effects diminished by inbreeding in Hippodamia convergens. Ecol. Entomol. 40, 596-602. 
519 [51] García-González, F. et al. (2015) Mating portfolios: bet-hedging, sexual selection and female multiple mating. Proc. R. Soc. Lond. B. Biol. Sci. 282, 20141525.

522 [52] García-González, F. et al. (2015) Risk-spreading by mating multiply is plausible and requires empirical attention. Proc. R. Soc. Lond. B. Biol. Sci. 282, 20150866.

[53] Holman, L. and Kokko, H. (2013) The consequences of polyandry for population viability, extinction risk and conservation. Phil. Trans. R. Soc. B. Biol. Sci. 368, 20120053.

[54] Price, T.A. et al. (2010) Polyandry prevents extinction. Curr. Biol. 20, 471-475.

529 [55] Greenway, E.G. et al. (2015) Mating failure. Curr. Biol. 25, R534-R536.

530 [56] Gerlach, N.M. et al. (2011) Promiscuous mating produces offspring with higher lifetime fitness. Proc. R. Soc. Lond. B. Biol. Sci. DOI:

533 [57] Klemme, I. et al. (2014) Polyandrous females produce sons that are successful at post-copulatory competition. J. Evol. Biol. 27, 457-465.

535 [58] Henshaw, J.M. and Holman, L. (2015) Bet-hedging via polyandry: a comment on 'Mating portfolios: bet-hedging, sexual selection and female multiple mating'. Proc. R. Soc. Lond. B. Biol. Sci. 282, 20150346.

538 [59] Holman, L. (2016) Bet hedging via multiple mating: A meta-analysis. Evolution, 70, 62-71.

540 [60] Maklakov, A. A. and Arnqvist, G. (2009) Testing for direct and indirect effects 541 of mate choice by manipulating female choice. Curr. Biol. 19, 1903-1906. 
542 [61] Fricke, C. et al. (2009) The conditional economics of sexual conflict. Biol. Lett.

$543 \quad 5,671-674$.

544 [62] Sirot, L.K. et al. (2015) Sexual conflict and seminal fluid proteins: a dynamic

545 landscape of sexual interactions. Cold. Spring. Harb. Perspect. Biol. 7, 017533.

546 [63] Bilde, T. et al. 2009. Postmating sexual selection favors males that sire

547 offspring with low fitness. Science. 324, 1705-1706.

548 [64] Simmons, L.W. (2001) Sperm competition and its evolutionary consequences

549 in the insects. Princeton University Press.

550 [65] Abe, J. and Kamimura, Y. (2015) Sperm economy between female mating

551 frequency and male ejaculate allocation. Am. Nat., 185, 406-416.

552 [66] Williams, P.D. et al. (2005) The evolution of sperm-allocation strategies and

553 the degree of sperm competition. Evolution, 59, 492-499.

554 [67] Fromhage, L. et al. (2008) Sperm allocation strategies and female resistance:

555 a unifying perspective. Am. Nat. 172, 25-33.

556 [68] Vahed, K. et al. (2011) Larger testes are associated with a higher level of

557 polyandry, but a smaller ejaculate volume, across bushcricket species

558 (Tettigoniidae) Biol. Lett. 7, 261-264.

559 [69] Vahed, K. and Parker, D.J. (2012) The evolution of large testes: sperm

560 competition or male mating rate? Ethol. 118, 107-117.

561 [70] Turnell, B. et al. 2018. Modelling strategic sperm allocation: Tailoring the 562 predictions to the species. Evolution. 72, 414-425.

563 [71] Byrne, P. et al. (2008) Effect of a persistent male courtship in the Drosophila 564 laboratory environment. Intgr. Comp. Biol. 48, e1. 
Glossary

567 Benefits driven polyandry: when females mate multiple times in order to gain direct

568 fitness benefits for themselves or indirect benefits that improve the fitness of their

569 offspring.

570 Convenience polyandry: when females mate more times than they need to achieve

571 full fertility because resisting extra mating attempts is more costly than acceptance.

572 Cost mitigation: any mechanism that serves to reduce loss of fitness caused by an

573 extrinsic stressor.

574 Costs of resistance (CR): fitness costs incurred by one sex as a result of attempting to 575 resist or avoid copulation.

576 Costs of mating (CM): fitness costs incurred by one sex as a result of copulation.

577 Direct benefits: when polyandry increases female longevity or fecundity, for

578 instance through nuptial gifts or ejaculate components.

579 Facultative female resistance (FFR): when females preferentially mate with males

580 that impose higher costs of resistance (CR), i.e. that are more coercive.

581 Fisherian benefits: when females gain a fitness benefit from mating multiply ('sexy

582 sperm') or with certain males ('sexy sons') because their sons are endowed with

583 their fathers superior competitive abilities under sexual selection.

584 Indirect benefits: when polyandry provides females with genetic benefits for their

585 offspring by improving offspring survival, fecundity and mating/fertilisation success.

586 Null polyandry: when female multiple mating serves to prevent reproductive failure.

587 Mate-guarding: after copulating one sex (usually the male) remains associated with

588 their partner to reduce the likelihood that they will mate again. 
Monandry: when females only mate once (strict monandry) or mate multiple times

590 with the same male (social monandry).

591 Polyandry: when females mate multiple times with multiple males.

592 Reproductive failure: when an individual fails to produce viable offspring either

593 because they fail to mate entirely, do not copulate with a compatible individual, or

594 do not receive or pass sperm during copulation.

595 Sexual harassment: when an individual of one sex attempts to mate with a non-

596 receptive individual of the opposite sex.

597 Sex-role reversal: a rare mating system characterised by paternal care, male choice

598 and competition between females over access to males. Commonly cited examples

599 include several species of pipefish and seahorse, wading birds such as phalaropes

600 and jacanas, and dendrobatid frogs.

601 Sexual receptivity: likelihood of an individual accepting a given mating attempt.

602 Sexual coercion: when an individual of one sex uses force or intimidation to copulate

603 with an individual of the opposite sex.

604 Sexual conflict: when the fitness optima for a trait differs between the sexes.

605 Superfluous mating: a mating that does not increase fertility or fitness.

606 Unconscious or implicit bias: beliefs about certain groups or concepts that

607 individuals are unaware that they possess.

610 Figure legends

611 Figure 1. How selection operates on the female mating rate under convenience

612 polyandry, benefits-driven polyandry, and null polyandry. Separating the costs of 
613 resistance $(C R)$ from the costs of mating $(C M)$ is crucial to understanding whether

614 polyandry is convenient. If $\mathrm{CR}$ and $\mathrm{CM}$ are grouped together then the forces that

615 select for polyandry (convenience vs benefits vs mating failure) are obscured. In this

616 figure we show how different forms of polyandry can result in selective feedback

617 loops between sexually selected male traits (grey boxes) and the female mating rate.

618 For instance, convenience polyandry and null polyandry generate selection on males

619 that may facilitate benefits-driven polyandry. Likewise, if strategic ejaculate

620 allocation renders females sperm depleted, there will be positive selection on the

621 female mating rate (to reduce MF). These feedback loops suggest that true

622 convenience polyandry is unlikely to persist for long, given the opportunities for

623 other forms of selection that it generates.

624

625

626 Figure 2. Distinguishing convenience polyandry and null polyandry [33]. Shown are

627 the predicted number of matings ( $A$ - solid lines) and proportion of mating attempts

628 accepted ( $B$ - dashed lines), when harassment (or encounters with males) varies.

629 Null polyandry (blue) occurs when females are equally receptive to each mating

630 attempt, and convenience polyandry (red) occurs when females change their

631 receptivity to mating according to the costs of resistance. These two cases are all but

632 indistinguishable with regards to mating number. When the proportion of mating

633 attempts accepted is considered however, we see that null polyandry (blue dashed

634 lines) does not change with the level of harassment, while for convenience

635 polyandry (red dashed lines) the proportion accepted increases. 
Box 1. A convenient case study - the water striders.

640 The water striders (Gerridae) (Figure I) are one group where the importance of

641 convenience polyandry (CP) has been empirically demonstrated [28]. The work

642 conducted in this family serves as a gold standard for understanding the importance

643 of cost mitigation for female mating rates. Yet these studies also show the

644 complexity of convenience polyandry, including the specific environmental

645 conditions required for it to occur, and the roles of female condition and mate

646 choice. Below we outline the work that has been done in the Gerridae, using the

647 framework we propose in Table 1 as a template.

648

649 In the Gerridae, females alter the proportion of matings they accept (criterion 1)

650 according the level of harassment they experience (the operational sex ratio; OSR

651 [28]). Mating is not without its costs for female water striders, and so they are often

652 reluctant to mate, which results in pre-mating struggles. These struggles (i) prevent

653 females from foraging efficiently and (ii) attract predators [28-29]. When females

654 permit copulation these costs are lowered because in some species females can (i)

655 forage efficiently whilst in copula. Predation risk (ii) is also lower when copulating

656 rather than struggling as the water surface is less agitated. The costs of mating vs

657 resistance are highly context dependent however, such that when females are

658 satiated or predation risk is removed mating is more costly than resistance $(C M>C R$,

659 criterion 2). Females respond to these context dependent costs, engaging in higher

660 levels of polyandry when it is convenient and resisting superfluous matings when it is 
661 not (criterion 3; [28]). The story becomes even more complex when we consider

662 facultative female reluctance (FFR; criterion 4), which occurs for instance in the

663 water strider Aquarius remigis. In A. remigis, females mate preferentially with large

664 males [30], but this only occurs when the costs of resistance are low compared to

665 the costs of mating (i.e. under low male density or when females are satiated; [28]).

666 When male density is high or females are hungry, convenience polyandry takes over

667 - the costs of resistance exceed the costs of mating with males regardless of size, and 668 females mate indiscriminately (criterion 4).

669

670

Box 2. Preconceptions about polyandry

671 In the sexual selection and sexual conflict literature, lingering historical

672 preconceptions about how females (including women) should behave have been

673 repeatedly suggested to colour our interpretations of male and female mating

674 behaviour in the absence of theoretically sound reasoning [45-46]. Societal norms

675 may be projected onto study species, with the sexes described using different

676 language even when the same behaviour is being studied. The role of socio-cultural

677 biases is perhaps most apparent in the case of sexual cannibalism, where loaded

678 terms with highly negative connotations (such as "rapacious", "voracious"; 48) are

679 used to describe females that behave in ways that are not in-line with societal

680 expectations.

681

682 Polyandry may represent another area where socio-cultural unconscious biases have

683 influenced interpretations of female mating behaviour (45). The lack of thorough

684 theoretical and empirical scrutiny that the convenience polyandry hypothesis has 
received, which contrasts markedly with the benefits-driven and bet-hedging

686 approaches [16-22], leads us to question why convenience is commonly described as

687 an explanation for female multiple mating. We suggest that socio-normative biases

688 about female behaviour may contribute to this pattern. When polyandry arises

689 through selection on males, as is the case for convenience polyandry, it fits with

690 societal expectations exemplifying the stereotype of the coy female responding to

691 the evolutionary demands of males: females only mate multiply to curtail the costs 692 imposed upon them by males.

693

694

695 


\begin{tabular}{|c|c|c|}
\hline & Criterion & Rationale \\
\hline 1 & $\begin{array}{l}\text { Females accept } \\
\text { a higher } \\
\text { proportion of } \\
\text { mating attempts } \\
\text { by males }\end{array}$ & $\begin{array}{l}\text { As harassment costs increase (i.e. the sex ratio } \\
\text { becomes more male-biased), females accept a } \\
\text { greater proportion of mating attempts. If only } \\
\text { the absolute number of matings is shown to } \\
\text { increase it is not possible to distinguish } \\
\text { between "null" polyandry and convenience } \\
\text { polyandry (Figure 2). If females increase the } \\
\text { proportion of mating attempts they accept, } \\
\text { then a case can be made for convenience } \\
\text { polyandry, because this suggests that females } \\
\text { alter their propensity to mate based on the } \\
\text { costs of resistance. }\end{array}$ \\
\hline 2 & $\begin{array}{l}\text { The costs of } \\
\text { resisting or } \\
\text { avoiding }(C R) \text { a } \\
\text { mating exceed } \\
\text { the costs of } \\
\text { mating }(C M)\end{array}$ & $\begin{array}{l}\text { Convenience polyandry cannot be assumed } \\
\text { when females mate multiply without gaining } \\
\text { any perceptible benefit. To demonstrate that } \\
\text { convenience polyandry may be operating } \\
\text { requires that CR exceed CM, i.e. resistance to } \\
\text { mating must be shown to carry greater fitness } \\
\text { costs than acceptance. }\end{array}$ \\
\hline 3 & $\begin{array}{l}\text { Females alter } \\
\text { their propensity } \\
\text { to mate } \\
\text { according to } \\
\text { changes in the } \\
\text { costs of } \\
\text { resistance (CR) }\end{array}$ & $\begin{array}{l}\text { Testing CR }>\mathrm{CM} \text { is not always straightforward as } \\
\text { context is often key. Moreover, when benefits } \\
\text { of polyandry accrue, } \mathrm{CM} \text { necessarily decreases } \\
\text { meaning that } \mathrm{CR} \text { may be relatively high without } \\
\text { polyandry being convenient. Therefore, it is } \\
\text { necessary to show not only that CR>CM } \\
\text { (criterion } 2 \text { ) but that females alter their } \\
\text { propensity to mate according to changes in CR. } \\
\text { Selection acts on the female mating rate } \\
\text { through different channels when polyandry is } \\
\text { convenient (reducing CR) compared to when it } \\
\text { is otherwise beneficial (reducing CM; see Figure } \\
\text { 1). }\end{array}$ \\
\hline 4 & $\begin{array}{l}\text { Females mate } \\
\text { indiscriminately } \\
\text { with respect to } \\
\text { male phenotype }\end{array}$ & $\begin{array}{l}\text { When polyandry is convenient the benefits that } \\
\text { females gain come from reducing CR not CM. As } \\
\text { such, under convenience polyandry the benefits } \\
\text { of accepting only preferred males are } \\
\text { superseded by the importance of mitigating the } \\
\text { overall costs of resistance. Cost mitigation can } \\
\text { also influence mate choice though because } \\
\text { individual males vary in how costly they are to } \\
\text { resist. Females may facultatively assess these } \\
\text { costs of resistance and alter their reluctance to } \\
\text { mate. While this appears to fit with the } \\
\text { definition of convenience polyandry, facultative } \\
\text { female reluctance (FFR) can confer indirect } \\
\text { benefits to polyandrous females. }\end{array}$ \\
\hline
\end{tabular}

\section{Examples}

The number of matings a female engages in increases with the sex ratio in the butterfly Bicyclus anynana. There are no data regarding the proportion of mating attempts that females accepted and so it is not possible to confirm convenience polyandry in this case.
$\mathrm{CR}>\mathrm{CM}$ can be tested using ablation studies, whereby females are maintained with males that have intact or ablated genitalia. If $C R$ $>\mathrm{CM}$ females maintained with ablated males will have lower fitness than those kept with intact males that can mate. Many ablation studies are not conducted under relevant ecological conditions and overlook key factors that make polyandry convenient (i.e. starvation, predation risk, Figure 1 and Box 1 ).

CR may not always exceed CM (see Box 1 ) and so ablation studies can yield contrasting results. The seed beetle, Callosobruchus maculatus offers a case study - females have been suggested to benefit from accepting superfluous matings because mateguarding reduces interruptions during oviposition. However, ablation studies do not support this assertion. This may be because mating is beneficial (CM drops) when females are dehydrated, as ejaculates are water rich. In order to test whether females respond to elevated CR (as opposed to reduced CM) in this species the benefits of mating must be removed (i.e. females should be hydrated). There are many other reported cases where female state can reduce $\mathrm{CM}$, and so context-dependent benefits should be considered when designing ablation studies.

Many examples exist where cost mitigation and mate choice are linked. For instance, females might mate preferentially with large males or males with large grasping apparatus. Examples such as these have sometimes been taken to suggest that the costs of resistance influence female mating decisions. The water striders (Box 1) offer a particularly good example of how these processes differ but can interact.

\section{Refs}

$[21,25,71]$

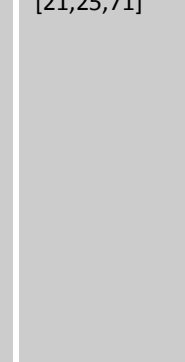

$[43,52]$

[53-57]

[58-59; 62]

an this Table we list the criteria that must be fulfilled to establish whether females mate multiply due to convenience polyandry, we explain our rationale, and we provide examples and methods to test each criterion. 

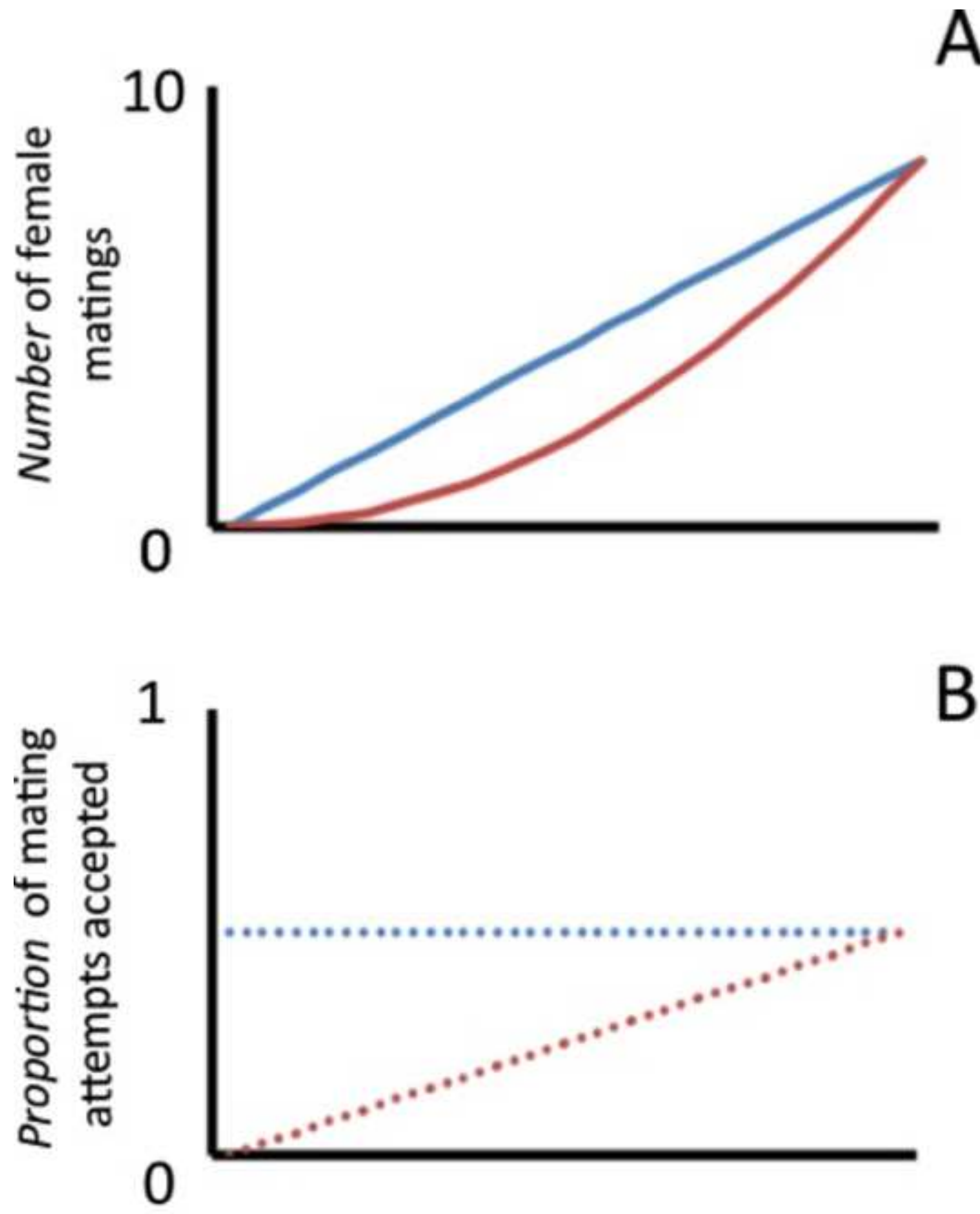

\section{Harassment / encounter rate}


Click here to access/download Original Figure File WS copulation for Box 1.jpg 


\section{Outstanding questions}

- Do existing examples of apparent convenience polyandry hold up to more rigorous testing under the framework that we propose?

- How common is convenience polyandry compared to benefits-driven polyandry?

- Direct and indirect benefits explanations for polyandry have been subject to considerable empirical and theoretical scrutiny - how does convenience polyandry compare when subject to similar assessment?

- Can/does convenience polyandry result in feedback loops that render multiple mating beneficial or necessary (rather than simply convenient)?

- Can polyandry evolve from a strictly monandrous state due to convenience?

- How has the social and historical context of sexual selection research influenced the way that we view and interpret polyandry? 